\title{
Valérie Nègre, L'art et la matière. Les artisans, les architectes et la technique (1770-1830)
}

Paris, Classique Garnier, coll. Histoire des techniques, 7, 2016

\section{Marie Thébaud-Sorger}

\section{OpenEdition Journals}

Édition électronique

URL : http://journals.openedition.org/artefact/1754

DOI : $10.4000 /$ artefact. 1754

ISSN : 2606-9245

Éditeur :

Association Artefact. Techniques histoire et sciences humaines, Presses universitaires du Midi

\section{Édition imprimée}

Date de publication : 30 mai 2018

Pagination : 301-304

ISBN : 978-2-7535-7494-6

ISSN : 2273-0753

\section{Référence électronique}

Marie Thébaud-Sorger, «Valérie Nègre, L'art et la matière. Les artisans, les architectes et la technique (1770-1830) », Artefact [En ligne], 7 | 2017, mis en ligne le 14 février 2019, consulté le 23 septembre 2020. URL : http://journals.openedition.org/artefact/1754 ; DOI : https://doi.org/10.4000/artefact. 1754

Ce document a été généré automatiquement le 23 septembre 2020.

\section{cc)}

Artefact, Techniques, histoire et sciences humaines est mise à disposition selon les termes de la Licence Creative Commons Attribution - Pas d'Utilisation Commerciale - Pas de Modification 4.0 International. 


\title{
Valérie Nègre, L'art et la matière. Les artisans, les architectes et la technique (1770-1830)
}

\author{
Paris, Classique Garnier, coll. Histoire des techniques, 7, 2016
}

\author{
Marie Thébaud-Sorger
}

\section{RÉFÉRENCE}

Valérie Nègre, L'art et la matière. Les artisans, les architectes et la technique (1770-1830), Paris, Classique Garnier, coll. Histoire des techniques, 7, 2016, 324 p.

1 L'ouvrage de Valérie Nègre est de ceux qui comptent pour l'histoire de l'architecture mais aussi pour l'histoire intellectuelle, sociale et culturelle des savoirs techniques au tournant des XVIII ${ }^{\mathrm{e}}$ et $\mathrm{XIX}^{\mathrm{e}}$ siècles. Son objet principal est d'éclairer le milieu des artisans et des architectes constructeurs français pour repenser à la fois les relations entre ces catégories de praticiens, mais ce faisant, pour mettre également en lumière la place centrale des techniques dans la construction de sujets d'intérêt général à un moment où s'affirment les préoccupations de salubrité, de sûreté, et de confort qui façonnent l'amélioration urbaine à la fin du siècle des Lumières.

2 L'étude de Valérie Nègre est organisée autour de trois grandes parties. La première est consacrée aux « décloisonnement des arts ", et saisit la participation de ces praticiens aux sociétés nouvellement créées que sont le Salon de la Correspondance générale sur les sciences et les arts fondé par Claude Pahin de la Blancherie, la Société libre pour l'encouragement des arts, métiers \& inventions utiles (1776-1781) de l'abbé Baudeau, puis sous la Révolution, la Société des inventions et découvertes (1791), la Société du point central des arts et métiers (1791) et le Lycée des arts (1792). Ces sociétés s'inscrivent dans le paysage de la promotion de la technique à la fin de l'Ancien régime et pendant la Révolution. Les examiner du point de vue des praticiens de l'architecture permet d'éclairer à nouveaux frais des thèmes centraux qui y sont alors débattus en vertu de 
l'utilité publique, initiant une réflexion partagée sur les qualités intrinsèques des matériaux (étanchéité, incombustibilité) et les formes de construction visant la robustesse et la simplicité d'usage. À travers ces différentes sociétés, l'auteure esquisse une galerie de portraits contrastés et montre comment ces hommes, en combinant plusieurs modes d'approche (la conversation, le concours, la discussion, l'évaluation, les démonstrations, l'exposition de modèles et la circulation d'imprimés), construisent différents cercles d'audiences et consolident des patronages princiers, ou avec des hommes de lois et d'État - particulièrement actifs dans les sociétés prérévolutionnaires. Ces lieux alternatifs offrent aussi une solution de substitution, parfois vécue comme une concurrence pour les institutions normatives comme l'Académie d'architecture, à la porte de laquelle nombre d'entre eux demeurent, puis sous la Révolution le bureau de consultations des arts et métiers.

La seconde partie, "Réinventer les matériaux », inscrit de façon neuve la recherche sur la matière au cœur de l'ouvrage. Valérie Nègre poursuit l'enquête sur les pratiques en saisissant l'activité inventive autour de trois études de cas : le carton, l'argile-marbre (pâte céramique maillée) et le ciment. Leur élaboration fait écho certes aux attentes d'utilités sociales et d'économie, mais elle souligne aussi en contrepoint un déplacement qui s'opère, induisant un nouveau régime esthétique de la matière libérée de la forme, où le goût du factice, porté par des élites avides de nouveautés, séduit et se diffuse largement. Cette partie réunit l'étude des promoteurs de ces procédés et celle du succès auprès de différents publics. L'importance de la commande aristocratique s'avère un phénomène déterminant des rouages de l'innovation. Même si on aurait aimé en savoir un peu plus sur les composants, les substances et les techniques employés, s'attacher à la relation entre nature et technique par ce prisme du travail sur la matière, permet à l'auteur de mettre en évidence quelques processus majeurs. Ainsi, loin de chercher à reproduire ou révéler la nature, les techniques cherchent ici à la surpasser (p.116). Si les inventeurs insistent sur la simplicité de procédés parfois inspirés de pratiques populaires ou utilisés par les anciens (on pense aux maisons « en pisé » de François Cointereaux ${ }^{1}$ ). L'usage de ces matériaux nouveaux prend la forme de réalisations audacieuses: chaises en cartons, four faïencé sans jointures apparentes, toits terrasses... Tout en réinscrivant leur recherche dans une dimension d'économie globale, le travail inventif de ces praticiens propose une « dénaturation » de la matière doublée d'une approche esthétique de transformation radicale qui se décline en autant d'oxymores surprenants tels que l'argile-marbre, le papier-pierre, ou la pierre-coulée (p. 154).

4 La troisième partie s'inscrit dans le renouveau historiographique autour du livre technique, auquel l'auteur a de longue date contribué et par lequel elle met en lumière plusieurs communautés de praticiens de l'architecture. Elle reprend ici une enquête fouillée comparant les deux grandes entreprises éditoriales techniques du siècle, $L a$ description des arts et métiers (1761-1788) et l'élaboration des articles et des planches de l' Encyclopédie (1751-1772). On voit se distinguer ici un groupe d'intermédiaires, artisans maniant l'art de construire et sachant dessiner dont les talents sont requis par les savants (p.169) mais qui sont habités par des modes de pensée, d'exposition et d'expression singuliers. Le corpus moins connu des traités de toisé de maçonnerie et d'arpentage, pourtant essentiel pour d'autres groupes de praticiens comme les expertsjurés, fait ensuite l'objet d'une étude. Combinant des connaissances techniques, mathématiques et juridiques ces ouvrages permettent d'établir des devis et de surveiller les chantiers. Ils visent ainsi à restreindre le contrôle de la construction 
ordinaire restée aux mains des maîtres de métiers à une période à la fois de reprise de l'activité constructive urbaine privée et d'une attention accrue des dépenses publiques de la part de l'administration des bâtiments du roi. On retiendra la figure de l'architecte juré-expert Nicolas le Camus de Mézières, célèbre pour la réalisation de la Halle au blé, dont Valérie Nègre étudie ensuite le traité dans un dernier chapitre au côté des publications de Pierre Patte et Jean Rondelet. Ces derniers exemples montrent comment leurs écrits à caractère scientifique sont fondés sur un travail de codification d'opérations abstraites, des expériences systématiques (essais de résistance de matériaux), des mises en parallèle historiques et critiques: ils monumentalisent et esthétisent la construction tout en déployant une technologie du bâti, distincte de celle décrite par les maîtres de métiers comme des approches intellectuelles de l'architecture savante (p. 216). Cette approche connaît un aboutissement institutionnel avec le cours de construction autonome créé en 1806 par Jean Rondelet.

5 Travailler sur l'identité sociale de ces praticiens est une tâche ardue mais dont on ne peut faire l'économie si l'on souhaite comprendre les phénomènes transversaux qui sous-entendent l'activité inventive entre les métiers, les procédés, les objets. Cependant, il ne s'agit ni de construire une prosopographie, ni de s'attacher à un groupe social bien identifié, homogène, à l'exemple des architectes-artistes ou des artisans (rattachés au corps de métiers). Au contraire, Valérie Nègre s'attache à dépeindre des espaces sociaux aux frontières plastiques qui recouvrent en partie toutes ces catégories socio-professionnelles sans leur être toutefois réductibles. Plus précisément, en choisissant le critère de la publicité, c'est-à-dire la manière dont ces acteurs identifient eux-mêmes leur activité, participent à l'espace public par leurs écrits, enseignements et expériences, et sollicitent les expertises et les publics, Valérie Nègre réunit un ensemble de figures types lui permettant de saisir les dynamiques à l'œuvre qui transforment l'art de bâtir: architecte-constructeur, artiste-artisan, expert-juré - une catégorie rassemblant architectes et entrepreneurs - témoignant de la porosité des frontières entre les activités (p.60), entrepreneurs (au sens donné par Hélène Vérin), risquant leur fortune dans des démarches aventureuses et dont la pratique est avant tout d'opérer « une médiation entre différents métiers et différents milieux sociaux » (p. 118), enfin amateurs, soucieux du bien commun.

Étudier ces catégories d'acteurs renouvelle l'étude des clivages existants entre monde académique et monde artisan. Si ce dernier a fait l'objet d'une réhabilitation dans l'historiographie cette dernière décennie, l'ambition est ici de dépasser la question de la valorisation de savoir-faire tacite, incorporé, pour étudier les savoirs véhiculés et construits entre sens pratique et inventivité technique. Or, la reconnaissance du "génie " de l'artisan portée par le mouvement encyclopédiste réhabilitant les arts et métiers s'avère un discours à double tranchant: non seulement son usage, qui se prolonge comme l'auteure le montre très bien dans l'héroïsation des figures de l'artisan au XIX ${ }^{e}$ siècle, s'opère en partie aux dépens de la reconnaissance de ce monde hybride des techniciens inventifs, mais aussi en vient in fine à dépouiller l'activité artisanale de son épistémologie propre en méconnaissant non seulement le caractère particulier mais aussi la dimension intensément collective. Pour ces praticiens qui revendiquent leur capacité à formaliser et mettre en ordre les savoirs, les tensions sont palpables au quotidien : Fourneau se voit contredit dans sa manière de présenter les relations entre pratique et théorie par Duhamel du Monceau pour l'édition de l'art du trait de charpente (p. 179) tandis que Loriot est traité de «faiseur de mastic » par le premier architecte du roi Richard Mique alors qu'il travaille à la restauration des voûtes de l'orangerie de 
Versailles (p.142-143). Une partie de l'Académie des sciences vient cependant à la rescousse de Loriot, rappelant ici qu'au sein des institutions d'État les positionnements sont loin d'être consensuels.

7 Enfin, Valérie Nègre met l'accent tout au long de l'ouvrage sur la constitution d'un espace public de la technique dans la société française de la fin du XVIII ${ }^{e}$ siècle. Celui-ci se façonne en premier lieu par la circulation des écrits. Il est également porté par une vague de publications éphémères de tout format, périodiques, annonces, affiches, gravures. Comme il a déjà été souligné, ce monde de l'imprimé renvoie matériellement à de multiples lieux: visites d'ateliers, informations sur les cours, annonces d'expériences. Les sociétés d'améliorations publicisent leurs réunions, expositions et appels à concours, s'adressant ainsi à des publics débordant les espaces de la légitimation académique et des communautés de métiers. Au cœur de ces interactions, une culture esthétique et singulière de la technique s'affirme, comme le montre par exemple la place des modèles dans ces dispositifs. Sur cet aspect majeur, l'ouvrage apporte un éclairage substantiel et bienvenu qui vient compléter d'autres travaux ${ }^{2}$. Ainsi les modèles - en nombre dans les cabinets de curiosité, mais souvent moins étudiés que les naturalia - constituent des éléments très attractifs parmi les pièces exposées au regard des amateurs du Salon de la correspondance des arts. Ils revêtent différentes formes: maquettes, modèles réduits requis lors de concours, fragments d'architecture ou dispositifs partiels, leur usage au cœur de la communication des savoirs et de leur apprentissage est attesté dans divers contextes d'enseignement.

8 En définitive, si l'enquête demeure centrée sur le milieu français, et particulièrement parisien, Valérie Nègre propose un ouvrage riche pour étudier, via l'architecture, le moment de fort décloisonnement des arts qui s'accompagne d'une rigidification des frontières entre les professions de la construction. Les écarts entre les fragments rassemblés sur les vies de ces acteurs, pour certains peu connus, et les récits qu'ils construisent à l'attention des élites et des publics, nous invitent à regarder en profondeur ces processus qui recomposent leurs pratiques et les savoirs, apportant de ce fait des éléments clés au dossier de l'épistémologie artisanale et de la formalisation de la technique. L'auteure nous invite de surcroît à envisager la spécificité de l'esthétique de l'expérimentation technique au XVIII siècle (p.155), ce goût pour l'artifice qui captive de larges audiences mais qui pour autant articule aussi l'exploit à des multiples attentes d'efficacité et d'utilités pratiques.

\section{NOTES}

1. Laurent BARIDON, Jean-Philippe GARRIC et Gilbert RICHAUD (dir.), Les leçons de la terre. François Cointeraux (1740-1830). Professeur d'architecture rurale, Paris, INHA/Éditions des Cendres, 2016.

2. Entre autres Celina fox, The Arts of Industry in the Age of Enlightenment. The Paul Mellon Centre for Studies in British Art, Yale, Yale University Press, 2010; Kelly Joan WHITMER, The Halle Orphanage as Scientific Community: Observation, Eclecticism and Pietism in the Early Enlightenment, 
Chicago, University of Chicago Press, 2015 ; Sabine FROMMEL, La Maquette d'architecture. Un outil au service du projet architectural, Paris, Cendres, 2015.

\section{AUTEURS}

MARIE THÉBAUD-SORGER

CNRS/Maison Française d'Oxford 